genes sit within the clusters. Although studies of fruitflies established the rules of spatial collinearity, this insect shows little, if any, evidence of temporal collinearity. On the other hand, evolutionarily more primitive insects appear to maintain temporal collinearity in Hox-gene expression. These findings tend to support the idea that temporal collinearity does require an intact Hox complex - although this may not be the only selective force holding the cluster together.

Another interesting lesson is emerging from comparisons of fruitflies, C. elegans, Ciona and Oikopleura with animals such as humans and mice. The former group of species make excellent experimental models, because of their rapid development and small genomes. But it is curious that the presumed ancestral bilaterian Hox complex has been split to varying degrees in each of these model systems - and temporal collinearity no longer applies. It might be that other aspects of their development and genome organization also differ substantially from their slower-growing and relatively largegenomed relatives, including humans. To be fair, however, the conclusion will probably be that every animal has its own unique and fascinating properties.

Nipam H. Patel is in the Departments of Molecular and Cell Biology, and of Integrative Biology,

University of California at Berkeley, Berkeley,

California 94720-3140, USA.

e-mail:nipam@uclink.berkeley.edu

1. Seo, H.-C. et al. Nature 431, 67-71 (2004).

2. Patel, N. H. \& Prince, V. E. Genome Biol. 1, reviews1027 (2000).

3. Kmita, M. \& Duboule, D. Science 301, 331-333 (2003).

4. van der Hoeven, F., Zakany, J. \& Duboule, D. Cell 85, 1025-1035 (1996).

5. Seo, H.-C. et al. Science 294, 2506 (2001).

Statistical physics

\title{
Hear the noise
}

\section{Šimon Kos and Peter Littlewood}

At the nanoscale, thermal fluctuations and noise dominate. But instead of being a hindrance, the details of the noise itself can reveal the physical properties of the system.

A lmost two centuries ago, the atomic nature of matter was elegantly revealed by brownian motion - as exemplified by the random motion of pollen particles in water as they are bombarded by water molecules. Now Crooker $e t$ al. ${ }^{1}$ provide, on page 49 of this issue, a textbook demonstration of fluctuations at work in the spectroscopy of a small number of alkali atoms - a quantum version of brownian motion.

In 1905, Einstein ${ }^{2}$ pointed out a subtle consequence of the fluctuations in classical brownian motion: the same random forces that make a pollen particle jitter would also cause friction if the particle were dragged through the water. In other words, the fluctuation of the particle at rest has the same origin as the dissipation of the motion of a moving particle that is subject to an external force. Einstein's result is a general one, codified in the 'fluctuation-dissipation theorem', which is one of the deepest results of thermodynamics and statistical physics. Einstein's observation also had a crucial consequence for the state of thermal equilibrium, in which the fluctuation of the stationary particle is characterized by a single number (the diffusion constant), and the friction of a moving particle is characterized by another number (the mobility). The fluctuation-dissipation theorem states that these two numbers, previously considered independent of each other, are, in fact, connected by a simple relation: they are proportional to each other through the absolute temperature, a characteristic of the state of thermal equilibrium.

Brownian motion follows Newton's classical equations. However, the invention of quantum mechanics, and the discovery of Heisenberg's uncertainty principle, brought discrete energy levels and new kinds of fluctuation into play. It took several decades to develop the proper techniques of quantum statistical physics, and the fluctuation-dissipation theorem did not reach its final form until the work of Kubo in the 1950s. Its formal derivation for quantum systems is even more mathematical and less transparent than in the classical case; many of us struggled to understand the physical context of this elegant but esoteric mathematical statement when we first met it in an undergraduate course. But the final result again has a clear physical interpretation: the dissipation in a quantum system is caused by transitions between its energy levels; the noise spectrum should, therefore, have peaks at frequencies corresponding to the differences in energy between these levels. All of the physical properties of a quantum system depend on the values of these energylevels: the 'colour' of an atom and its nuclear magnetic resonance frequency are two examples that involve measuring the energy spectrum.

Quantum physics began with the study of the emission lines of the hydrogen atom the particular wavelengths of radiation emitted as the atom's electron makes transitions between energy states. In their experiment,
Crooker et al. ${ }^{1}$ study the magnetic fluctuations in vapours of hydrogen-like alkali atoms of rubidium and potassium. These atoms have a single valence electron, whose direction of spin, or magnetization, can change. Crooker et al.measure birefringence, a phenomenon well known in solutions of helical organic molecules such as sugars: because of the helicity of the molecules, right- and left-polarized light propagates through the solution with different speeds, and hence incident linearly polarized light leaves the sample with its direction of polarization rotated.

The same effect happens for alkali atoms in their ground state where the helical orientation is provided by the spin of the valence electrons. Crooker and colleagues' experiments show that the polarization of light fluctuates in time, as the spin magnetization itself fluctuates. But rather than following the maxwellian distribution of classical thermal noise, the temporal fluctuations have a complex spectral structure - hyperfine structure - owing to a delicate interaction between the spin of the valence electron and that of the atomic nucleus. These lines can be resolved, as predicted, despite being narrower than the linewidth of the probe laser. Noise produced by a summation of random events grows more slowly than the system size. Crooker et al. ${ }^{1}$ show that the noise per atom scales inversely as the square root of the number of atoms, as expected.

Often we study systems by perturbing them - by measuring their response to an external probe. But this approach becomes increasingly difficult for the small systems that are now the focus of many studies in nanoscale or biological sciences. These experiments ${ }^{1}$ remind us that 'listening' to the intrinsic noise of a system in equilibrium can provide the same information as does probing it with an external field (which in the present case would be equivalent to performing a conventional magnetic resonance experiment on the electron spins). Crooker et al. have provided an elegant example of a general principle, one that might be exploited, for example, in chemical sensors by measuring the thermal vibration of small cantilevers ${ }^{3}$, or could be used to measure the fluctuations of a single electron spin on a surface using a scanning tunnelling microscope $e^{4,5}$. For the moment, however, it is a practical demonstration of an arcane yet fundamental piece of science, first intuited by Einstein during his annus mirabilis a century ago.

Simon Kos and Peter Littlewood are in the Theory of Condensed Matter Group, Cavendish Laboratory,

Madingley Road, University of Cambridge,

Cambridge CB3 OHE, UK.

e-mail:pbl21@cam.ac.uk

1. Crooker, S. A., Rickel, D. G., Balatsky, A. V. \& Smith, D. L. Nature 431, 49-52 (2004).

2. Einstein, A. Ann. Phys. 17, 549-560 (1905).

3. Paul, M. R. \& Cross, M. C. Phys. Rev. Lett. 92, 235501 (2004).

4. Balatsky, A. V. et al. Phys. Rev. B 66, 195416 (2002).

5. Rugar, D., Budakian, R., Mamin, H. J. \& Chui, B. W. Nature 430, 329-332 (2004). 